\title{
Buchbesprechungen - Books Review - Livres Nouveaux
}

\section{Monges, H.: Les stases gastriques. Doin, edit. Paris 1949. Preface de R. A. Gutmann.}

La question des stases gastriques est restée trop longtemps confondue avec celle des stenoses du pylore: quand $\Gamma$ estomac ne se vide pas dans les délais voulus, on admet implicitement qu'un fonctionnement défectueux, un spasme pour le moins, de ce sphincter, est en cause. Certes on connaît bien la paralysie aiguë de Гestomac, souvent post-opératoire, à allure dramatique par son evolution rapide et son pronos-tic severe. II existe cependant tout un groupe de faits cliniques dont la classique «indigestion», terminée par un vomissement libérateur est le type commun qui relèvent de la «paralysie aiguë simple», le plus souvent méconnue, car elle est transitoire, pauvre en symptômes cliniques, et échappent habituellement à $\Gamma$ examen radiologique, dont les constatations éventuelles sont d'ailleurs mal interprétées.

Aussi H. Monges rendra-t-il de grands services en établissant la frequence de ces faits et en apprenant à distinguer les dilatations par «asystolie gastrique», après épuisement de la musculature fatiguée de lutter contre un obstacle pylorique, de celles provenant d'une «atonie» subite, mais passagère, de cette même paroi musculaire de Гestomac.

Après avoir décrit les symptômes, communs à toutes ces stases gastriques et leur critère radiologique, M. rappelle, dans un court paragraphe, les notions classiques -et toujours valables sur les stenoses pyloríques; et, un peu plus longuement, celles concernant la dilatation aiguë de Testomac.

La partie originale de Гouvrage s'occupe de la paralysie gastríque aiguë simple, terme propose en 1934 par J. Monges pour designer un état si banal qu'il échappe au diagnostic du médecin, qui se méprend sur sa veritable nature. La clinique nous apporte en effet seulement de la gene épigastrique, une sensation de tension, quel-

\section{2}

\section{Varia}

ques eructations, le tout dure à peine quelques heures, puis tout rentre dans $\Gamma$ ordre, après ou sans vomissement. Si $\Gamma$ on fait avaler au malade de la baryte à ce moment, on constate avec étonnement rimage en «cuvette», en «assiette à soupe» de la grande sténose pylorique, avec atonie totale de l'estomac, et un pylore fermé; mais un contrôle éventuel, le lendemain, montrera un organe d'aspect radiologique tout à fait normal. La paralysie aiguë simple de Testomac reconnaît un grand nombre de causes. Elle peut être provoquée par une emotion ou correspond avec une lipo-thymie, mais elle survient aussi après une commotion cérébrale ou une migraine, dans les syndromes médiastinaux, le mégacôlon, Гappendicite chronique, certaines stenoses intestinales, affections rénales et maladies infectieuses (diphtérie notamment).

Le chapitre de pathogênie s'efforce de faire la liaison entre tous ces états: Testomac possède un automatisme neuro-moteur autonome, modifié par Гaction des nerfs vague et sympathique. La théorie mécanique («compas mésentêrico-duodénal») ne résiste pas à Гanalyse des faits; il est plus exact d'incriminer une paralysie de Гestomac (gastroplégie), le plus souvent par hypersympathicotonie. Cependant, il faut y ajouter un trouble de la perméabilité capillaire, pour expliquer Гénorme afflux de liquide - très supérieur aux possibilités des glandes sécrétoires - 
dans la cavité gastro-duodénale. II existe done un stade musculaire, puis un stade vaso-moteur, sans oublier la participation des facteurs nerveux et toxiques dans la haute gravité des dilatations aiguës. Dans les paralysies aiguës simples, si le pylore est fermé, e'est par excitation du splanchnique, nerf inhibiteur; ici e'est le phénomène nerveux réflexe qui paraît au tout premier plan; le point de depart de ces reflexes est avant tout intestinal (chronique ou mécanique). II est fort intéressant de rapprocher de ces faits certaines stases gastriques, transi-toires, réversibles, au cours des poussées douloureuses des ulcères, qu'il ne faut pas interpreter comme une sténose vraie, scléro-cicatricielle, mais qui est liée à une «stênose inflammatoire», ou à une «gastrite ectasiante». Enfin il faut encore classer ici les estomacs dilates (méga-estomacs de type paralytique).

Le chapitre de thérapeutique est court: il s'agit surtout de ne pas porter un faux diagnostic de sténose, avec la sanction chirurgicale qu'il comporte. Traitement sédatif, anti-inflammatoire chez les ulcéreux; infiltration anesthésiante ou resection des splanchniques chez les dilates; evacuation des liquides gastriques dans les paralysies aiguës - telles sont les mesures simples, mais dont Гapplication précoce évitera les evolutions catastrophiques. M. Demole

Varia

Hommage au Dr R. A. Gutmann

Les élèves du Dr. R. A. Gutmann, médecin des hôpitaux de Paris, auteur d'un traité classique (Syndromes douloureux de la region épigastrique), spécialiste uni-versellement connu des problèmes de Tulcère, ont decide de lui offrir une médaille pour lui témoigner leur reconnaissance à Гoccasion de son prochain depart de $\Gamma$ Hôpital St-Antoine.

Les inscriptions doivent être adressées au Dr. R. Dupuy, 244, Bd. Raspail, Paris XIV0. Tout souscripteur d'une somme minima de fr.fr. 2500.- recevra une reproduction de la médaille, dont la realisation a été confiée au Maître Auricoste. 